\title{
$\dot{E}$ LE C TR ICITÉ
}

\section{Comment choisir un régulateur automatique}

\author{
(SUITE)
}

Par E. JUILLARD, Ingénieur E. P. Z.

Appliquons maintenant les principes généraux qui viennent d'ètre rappclés à l'étude plus approfondie d'un régulateur : nous examinerons l'appareil à pression d'huile type "Rex ", construit par les Ateliers H. Cuénod, à Genève (Suisse). Cette étude comprendra :

$1^{\circ}$ La description de l'appareil ;

$2^{\circ}$ L'établissement de son équation propre;

$3^{\circ}$ L'application au cas du réglage de tension d'une génératrice, c'est-à-dire l'établissement des équations de la génératrice et de la correction ;

$4^{\circ}$ Le contrôle expérimental.

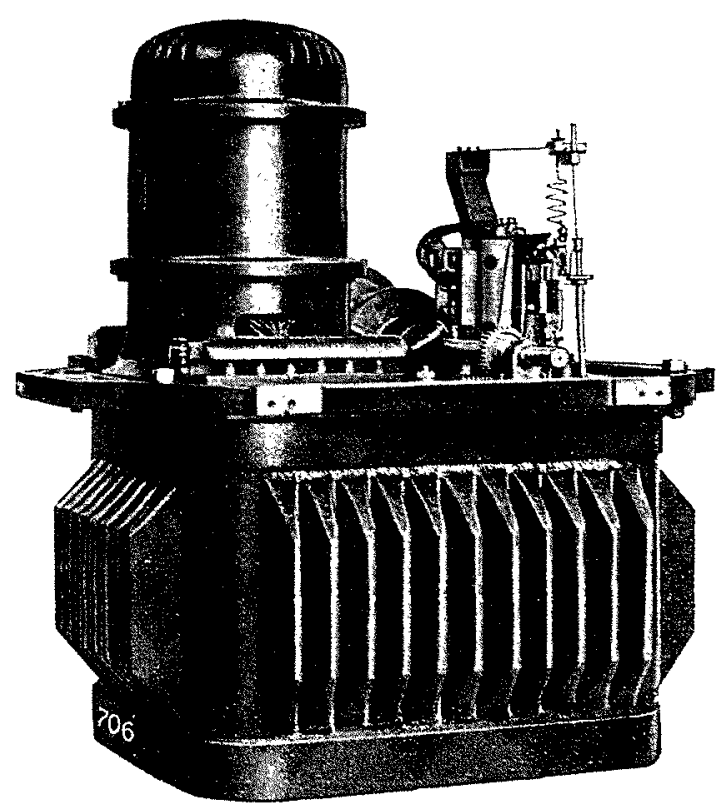

diaire d'un frein à huile 27 , transmet son mouvement à une lame élastique 24, à laquelle est attaché le ressort antagoniste 22 de l'appareil de mesure 13. Le ressort supplémentaire 23 n'a d'autre but que do permettre d'amener, par le moyen de la vis à poulets à laquelle il est attaché, le fléau de l'appareil de mesure dans sa position normale. Tout ce dispositif a pour but d'amortir les oscillations de l'appareil, c'est-à-dire de stabiliser le réglage.

Différentes dispositions particulières sont prises pour augmenter la sensibilité et la rapidité d'action de cet appareil sans nuire à sa robustesse :

10 Tout le mécanisme, sauf l'appareil de mesure, est contenu dans l'huile ; le moteur, placé verticalement, occupe la place de la boîte d'engrenages coniques, figurée dansle schéma (fig. 7);

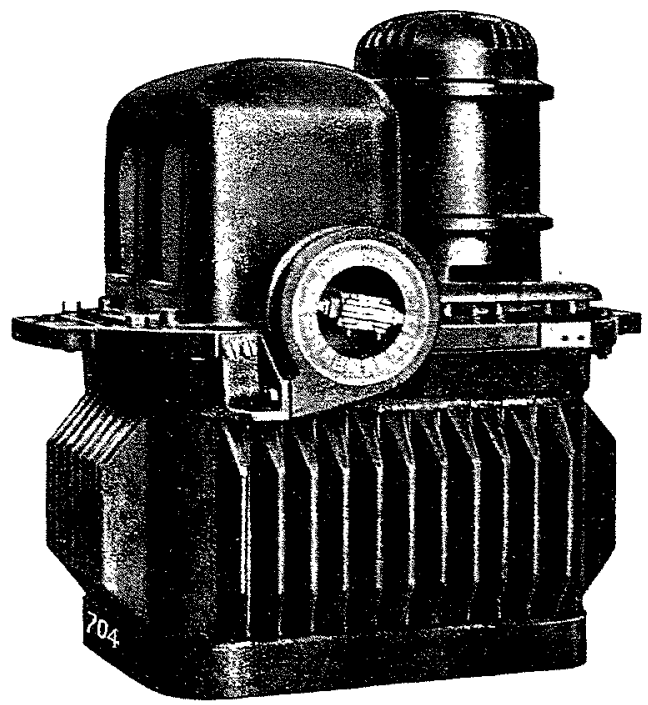

Fïg. 13.. - Régulateur à pression d'huile, type " Rex».

Description du Régulateur a Pression d'Huile Type «Rex»

Cet appareil est représenté schémaliquement par la fig. 7 (voir Nos 77-78 de mai-juin 1923). Une petite pompe centrifuge 2 , entretenue en mouvement permanent par moteur électrique, fournit de l'huile sous pression à un distributeur 3. Par le jeu du tiroir de ce distributeur l'huile est envoyée d'un côté ou de l'autre d'un piston rotatif 15 , qu'elle entraîne dans le sens voulu.

En régime stationnaire le piston, ou plus exactement le volet 15 , est immobile : les lumières sonl fermées des deux côtés. Mais lorsque par suite d'une variation de l'intensité circulant dans l'appareil de mesure 13 , le tiroir est déplacé verticalement, les lumières sont ouvertes du côté de l'admission d'une part, et du côté de l'évacuation d'autre part, et le volet est cntraîné.

Sur l'arbre 14 du volet est fixé un petit pignon qui entraîne dans son mouvement un secteur denté 25. Ce secteur, par liutermé- $2^{\circ}$ Le tiroir circulaire du distributeur est animé d'un mouvement de rotation autour de son axe, dans le but d'éliminer l'effet des frottements dans le sens vertical et de le faire obéir aux plus petits déplacements de l'appareil de mesure ;

$3^{\circ}$ Le tiroir 10 du distributeur n'est pas relié rigidement à l'appareil de mesure, mais par l'intermédiaire d'un petit tiroir auxiliaire 11, excessivement léger. Ce tiroir 11 commande, dans le grand tiroir 10, des lumières qui, grâce à l'huile sous pression, asservissent instantanément lès déplacements du grand tiroir à ceux du petit. L'appareil de mesure n'a donc pas d'organe lourd à entrâner et les déplacements du grand tiroir se font néanmoins avec toute la rapidité et la précision voulues, puisque l'énergie nécessaire est empruntée à l'huile sous pression ;

$4^{\circ}$ Enfin, par la rotation du grand tiroir, l'huile est envoyée périodiquement ct constamment, mais en petite quantité, d'un 
côté et de l'autre du volet 15. Celui-ci n'est done jamais immobile mais oscille, en régime stationnaire, autour d'une position moyenne. L'amplitude de cette oscillation varie selon le but que l'appareil a à remplir et la nature des organes entraînés. La fréquence en est assez élevée (10 à 20 périodes par seconde).

L'arbre de réglage 14 actionne les organes de réglage ou porte lui-même les frotteurs des appareils à touches. Dans le régulateur destiné spécialement au réglage d'excitatrice, l'appareil à touches est réalisé sous forme de collecteur avec balais en charbon. Le tremblotement dont il est question ci-dessus subdivise en quelque sorte la valeur de la résistance comprise entre deux touches consécutives et donne à l'appareil une sensibilité très grande (de l'ordre de grandeur de 2 à 3 pour mille). La fréquence de la vibration ne se remarque évidemment plus sur le courant d'excitation ; elle a en outre l'avantage d'ètre invariable.

\section{L'Equation de Régllateli}

Supposons que l'équipage mobile de l'appareil de mesure (fig.14) soit déplacé d'une valeur " $y$ " mesurée au point d'attache $A$ du tiroir. Comme, d'une parl, les sections de passage des lumières

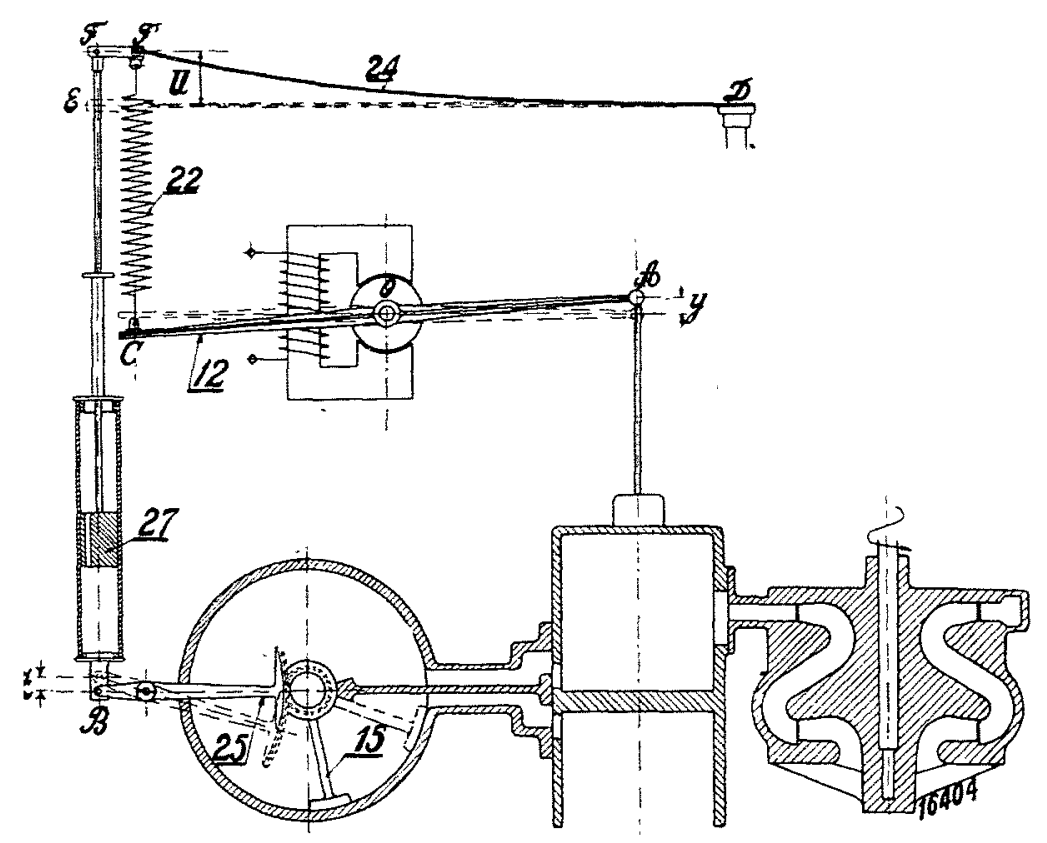

Fig. 14.

sont proportionnelles à ce déplacement et comme, d'autre part, la pompe centrifuge fournit une pression invariable dans de très larges limites, la quantité d'huile passant par unité de temps dans le tambour sera proportionnelle au déplacement $y$. La vitesse du volet 15, mesurée par le déplacement du point B du secteur 25 , sera proportionnelle à $y$ :

$$
\text { ) } \quad \frac{d z}{d t}==\mathrm{V} y
$$

$V$ désignant ce facteur de proportionnalité qui dépend uniquement de la pression de la pompe et des dimensions des canaux.

Sur l'équipage mobile 12 agissent deux efforts : le couple électromagnétique produit par le courant " $i$ " circulant dans l'enroulement et l'effort antagoniste du ressort 22 . $\mathrm{Si}$, ce qui est généralement le cas, l'effort électromagnétique est indépendant de la position de l'équipage mobile, celui-ci n'effectuant jamais de déplacement supérieur à $2^{\circ}$ autour de sa position moyenne, nous aurons entre ces deux efforts la relation d'équilibre : (A O étant égal à $\mathrm{OE})$.

$$
\mathrm{F}_{0}+\beta(u+y)=M i^{2}
$$

$F_{0}$ est la tension initiale du ressort $22, \beta$ en est le coefficient.

Supposons le régulateur en régime stationnaire et immobile et soit $i_{n}$ la valeur du courant correspondant à ce régime; $i_{n}$ est donc la valeur de consigne. L'équipage mobile doit alors occuper la position horizontale pour laquelle toutes les lumières du distributeur sont fermées : donc $y==o$. En outre, la lame d'asservissement sera détendue et occupera également la position D E, ne produisant plus aucun effort sur le piston du frein à huile 27 : donc ainsi $u=o$ et nous aurons $\mathrm{F}_{0}=\mathrm{Mi}_{\mathrm{u}}{ }^{2}$.

par soustraction $\quad \hat{s}(u+y)=\mathrm{M}\left(\mathrm{i}^{2}-i_{\mathrm{n}}{ }^{2}\right)$.

Si nous posons $\left.i=i_{\mathrm{n}}+\right\lrcorner i$ et que nous n'envisagions que des différences assez petites, le binôme $i^{2}-i_{\mathrm{n}}{ }^{2}$ peut se remplacer par $2 i_{\mathrm{n}} \perp$ et

$$
3(u+y)=2 \mu i_{\mathrm{n}^{2}} \frac{\Delta i}{i_{\mathrm{n}}}
$$

$\frac{\Delta i}{i_{\mathrm{n}}}$ n'est autre chose que l'ècart relatif de la valeur mesurée par l'appareil de mesure, c'est-à-dire la fonction de régime. En désignant cet écarl relatif simplement par $\Delta$, nous aurons done:

$$
3(u+y)=2 M i_{u}{ }^{2}
$$

Asservissement; Lorsque le volet 15, donl nous mesurons le déplacement par celui du point $B$, se dirige vers le haut, il entraine avec lui le piston du frein à huile et tend la lame 24 . La vitesse relative du piston 27 dans son cylindre est donc: $\left[\frac{d z}{d t}-\frac{d u}{d t}\right]$ et l'effort qui fait remonter le piston, proportionnel à celle vitesse, est équilibré par l'effort $\beta_{\mathrm{a}} u$ développé par la flexion de la lame d'asservissement. D'où la troisième relation :

ou

$$
\tilde{r}_{\mathrm{a}} u=\mathrm{A}\left[\frac{d z}{d t}-\frac{d u}{d t}\right]
$$

$$
\frac{d z}{d t}=\frac{d u}{\mathrm{~d} t}+\frac{u}{\mathrm{~T}_{\mathrm{a}}}
$$

Le rapport $\frac{A}{\beta_{a}}=T_{a}$ a la dimension d'un temps. Il représente la constante de temps de l'asservissement et dépend des propriétés élastiques de la lame 24 et du freinage du piston de la pompe 27.

En éliminant de ces 3 équations les.variables " $u$ " et " $y$ ", nous obtiendrons la relation cherchée entre l'écart relatif $\Delta$, le déplacement de l'arbre de réglage et le temps. Cette élimination nous donne :

$$
\frac{d \Delta}{d t}+\frac{\Delta}{T_{\mathrm{a}}}-\frac{\hat{\beta}}{2 \mathrm{M} i_{\mathrm{n}}^{2} \cdot \mathrm{V}} \frac{d^{2} z}{d t^{2}}+\frac{\mathrm{B}}{2 \mathrm{Mi}_{\mathrm{n}}^{2} \cdot i}\left[1+\frac{1}{\mathrm{VT}_{\mathrm{a}}}\right] \frac{d z}{d t}
$$

Les coefficients des variables et de leurs dérivées ont des significations simples.

Supposons l'asservissement supprimé, ce qui revient à supposer nul le frottement opposé au piston de la pompe.

Dans ce cas $T_{a}=0$ et notre équation se réduit à :

$$
\Delta=\frac{\beta}{2 M i^{2} \cdot \mathrm{V}} \frac{d z}{d t}
$$

Si dans cette hypothèse on maintient un écart $\Delta$ invariable, la vitesse de l'arbre de réglage sera constanto el égale à $: v=\frac{d z}{d t}=$ 
$\frac{2 M i^{2} n^{2} \mathrm{~V}}{\mathrm{~B}} \Delta$, c'est-à-dire en particulier proportionnelle à l'éc'art à corriger $\Delta$.

Si S est le parcours total de l'arbre de réglage, le temps qu'il faudra à l'arbre pour effectuer loute sa course dans ces conditions, sera :

$$
t=\frac{\mathrm{S}}{v}=\frac{\mathrm{S} \cdot \beta}{2 \mathrm{M} i_{\mathrm{n}}^{2} \cdot \mathrm{V} \Delta}
$$

d'où l'on lire :

$$
t . \Delta=\frac{\mathrm{S} \beta}{2 \mathrm{M} i_{\mathrm{n}}^{2} \cdot \mathrm{V}}=\mathrm{T}_{\mathrm{s}}
$$

Le produit $t . \Delta$ du temps nécessaire au régulateur pour accomplir toute sa course sous l'effel d'un écart constant, par la valeur relative de cet écart, abstraction faite de l'asservissement, est ce que nous avons désigné par durée spécifique de parcours. Cetle durée ne dépend que des caractéristiques de l'appareil. El nous pouvons remplacer la valeur

$$
\frac{\beta}{2 M i_{n}^{2} \cdot V} \operatorname{par} \frac{T s}{S}
$$

dont la signification est plus immédiate. L'équation devient :

$$
\frac{d \Delta}{d t}+\frac{\Delta}{\mathrm{T}_{\mathrm{a}}}=\mathrm{T}_{\mathrm{s}} \frac{d^{2} \frac{z}{\mathrm{~S}}}{d t^{2}}+\mathrm{T}_{\mathrm{s}}\left(\mathrm{V}+\frac{1}{\mathrm{~T}_{\mathrm{a}}}\right) \frac{d \frac{z}{\overline{\mathrm{S}}}}{d t}
$$

$\overline{\mathrm{S}}==$ est le parcours relatif, la course totale étant égale à 1 .

$$
\frac{d \Delta}{d t}+\frac{\Delta}{\mathrm{T}_{\mathrm{a}}}=\mathrm{T}_{\mathrm{s}} \frac{d^{2} \psi}{d t^{2}}+\mathrm{T}_{\mathrm{s}}\left(\mathrm{V}+\frac{1}{\mathrm{~T}_{\mathrm{a}}}\right) \frac{d \psi}{d t}
$$

Supposons enfin que l'asservissement soit rigide, ce qui revient à supposer la pompe à huile remplacée par une tige rigide, ou remplie d'huile infiniment visqueuse. $T_{a}$ devient alors égal à $\infty$, et

$$
\frac{d \Delta}{d t}=\mathrm{T}_{\mathrm{s}} \frac{d^{z_{u}}}{d t^{2}}+\mathrm{VT}_{\mathrm{s}} \cdot \frac{d \grave{y}}{d t}
$$

qui, intégrée, donne :

$$
\Delta=\mathrm{T}_{\mathrm{s}} \frac{d \psi}{d t}+\mathrm{VT}_{\mathrm{s}} \psi+\text { constante. }
$$

Examinons maintenant l'équilibre, c'est-à-dire les valeurs de $\Delta$ pour lesquelles la vitesse de manœuvre $\frac{d \psi}{d \dot{t}}=0$. Si tel doit être le cas, nous aurions $\Delta=\mathrm{VT}_{\mathbf{s}} \psi+$ constante, ce qui signifie que l'immobilité du régulateur n'est réalisée en chaque position (pour chaque valeur de $\psi$ ) que par une valeur bien déterminée de l'écart, différente de point en point. Si $\Delta_{1}$ et $\Delta_{2}$ sont les valeurs de l'écart rela tives aux deux extrémités de la course, nous aurions :

Pour

$$
\begin{array}{ll}
\psi=0 & \Delta_{1}=\text { constante } \\
\psi=1 & \Delta_{2}=V \mathrm{~T}_{\mathrm{s}}+\text { constante }
\end{array}
$$

d'où

$$
\Delta_{1}-\Delta_{2}=\delta=\mathrm{T}_{\mathrm{s}} \mathrm{V}=\frac{\mathrm{S} \beta}{2 \mathrm{Mi}{ }_{\mathrm{n}}^{2}}
$$

os est le décrément de l'asservissement, c'est-à-dire l'altération du point de réglage ou altération de la consigne qui résulterait si la poimpe d'asservissement était remplacée par une tige rigide. C'est, si l'on veut, l'altération momentanée ou passagère de la valeur de la consigne, altération dont le rôle est précisément de stabiliser l'opération de réglage.

L'évaluation de $\delta$ est des plus simples. $S \beta$ est la variation d'effort développée par le ressort de l'appareil de mesure, Iorsqu'il est tendu d'une valeur égale à la course totale $\mathrm{S}$, et $\mathrm{M} i_{\mathrm{n}}{ }^{2}$ est l'effort fourni par l'appareil de mesure lorsqu'il est parcouru par la valeur normale de consigne.

L'équation générale du régulateur s'écrit donc :

$$
\frac{d \Delta}{d t}+\frac{\Delta}{\mathrm{T}_{\mathrm{a}}}=\mathrm{T}_{\mathrm{s}} \frac{d^{2} \psi}{d t^{2}}+\left(\delta+\frac{\mathrm{T}_{\mathrm{s}}}{\mathrm{T}_{\mathrm{a}}}\right) \frac{d \psi}{d t}
$$

et contient 3 grandeurs caractéristiques :

10 La constante de temps d'asservissement $\mathrm{T}$; $2^{\circ}$ Le décrément d'asservissement $\delta$;

$3^{\circ}$ La durée spécifique de parcours $T_{\mathbf{s}}$.

L'équation ci-dessus traduit aussi le fonctionnement d'autres types de régulateurs. Elle est valable en particulier également pour :

Le régulaleur à déclic système Thury ;

Le régulateur à action directe système B. B. C. Baden ;

Le régulateur Thyrill, etc...

es cusiizizis ayant éviłamment dans chaque cas des valeurs ldifférentes.

Le degré d'insensibilité ne se traduit pas par cette équation. Comme il est en général dû à des frottements ou à des jeux d'organes, il se mesure directement; il n'a du reste pas d'influence appréciable sur la forme de la correction.

\section{Applicátion au gas D'un Réglage simple}

Avant d'entreprendre l'étude du réglage de la tension d'une génératrice, examinons le cas très simple du réglage de la tension d'une batterie d'accumulateurs. Cela nous permettra de fixer la suite du raisonnement.

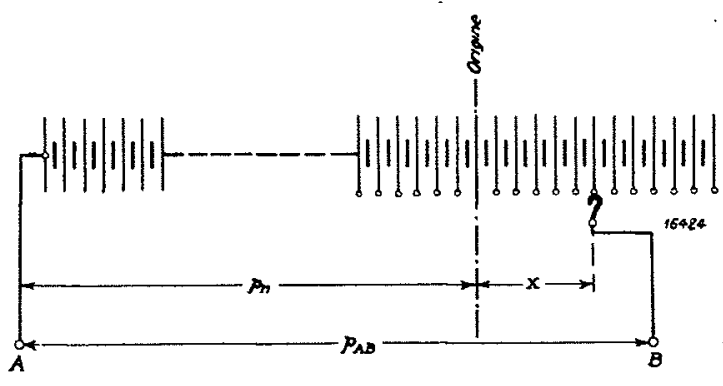

Fig. 15.

Supposons une batterie à très grand nombre d'éléments et remplaçons, par la pensée, les forces électromotrices des éléments réels par une suite de forces électromotrices infiniment petites, dont la somme reste égale à la force électromotrice de la batterie. Cela revient à supposer que la tension entre $A$ et $B$ varie d'une manière continue avec le déplacement $x$ du curseur, proportionnel au nombre d'éléments insérés.

$x$ est le paramètre de réglage.

Equation du système. - La tension A B peut alors s'écrire:

$$
p_{\mathrm{AB}}=p_{\mathrm{n}}+M x
$$

L'origine des déplacements est placée à l'endroit où la tension a la valeur normale $p_{n}$.

Si nous faisons usage d'un régulateur automatique, nous commanderons le déplacement du curseur B par l'arbre de réglage. 
Le déplacement du curseur étant établi de façon à rester proportionnel à la course du régulateur, nous pouvons donc aussi poser :

$$
p_{\mathrm{AB}}=p_{\mathrm{n}}+m\left(\psi-\psi_{0}\right)
$$

Un écart $\lrcorner p=p_{\mathrm{AB}}-p_{10}$ d'avec la normale sera donc représenté par $\Delta p=m\left(\dot{\psi}-\psi_{0}\right)$. Et l'écart relatif

$$
\Delta=\frac{\Delta p}{p_{\mathrm{n}}}=\frac{m}{p_{\mathrm{n}}}\left(\psi-\psi_{0}\right)=-\gamma\left(\psi-\psi_{0}\right)
$$

$\gamma$ est le coefficient de répartition sur le cadran.

L'écart est nul dans la position $y_{0}$.

En déplaçant le curseur d'une extrémité $(\psi=0)$ à l'autre $(\downarrow=1)$ de sa course, on produit donc une variation totale de la tension égale à :

$$
\Delta_{\text {total }}=-\gamma
$$

Le coefficient de répartition est done l'écart relatif total obtenu en déplaçant le curseur d'une extrémité à l'autre de sa course.

Régulateur. $=$ Nous ferons usage d'un régulateur sans asservissement. On peuts'attendre à ce que le réglage soit néanmoins stable dans ce cas, parce que l'équation de la batterie ne contient pas le temps explicitement. En faisant donc $T_{a}=O$ dans l'équation du régulateur, on obtient ici :

$$
د=\mathrm{T}_{\mathbf{s}} \frac{d \downarrow}{d t} \text { équation du régulateur. }
$$

que nous devons donc combiner avec l'équation de la batterie

$$
\Delta=-\gamma\left(\dot{\psi}-\psi_{0}\right)
$$

La résolution de ces deux équations par rapportà donnera les équations de la correction, c'est-à-dire les déplacements en fonction du temps; et l'allure de l'écart pendant la correction.

La solution se trouve aisément :

$$
\begin{gathered}
y-y_{0}=\text { constante } \cdot e^{-\frac{\gamma}{T_{s}} t} \\
\Delta=-\gamma \cdot \text { constante } \cdot e^{-\frac{\gamma}{T_{s}} t}
\end{gathered}
$$

La constante d'intégration s'évalue par la condition limite au départ. Nous supposons qu'au moment où le régulateur a eu à intervenir, l'écart avait une valeur initiale $\Delta_{i}$. Le curseur se trouvant alors en un point $\psi_{\mathrm{i}}$, lié à $\Delta_{\mathrm{i}}$ parl'équation du système 8)

$$
\Delta_{\mathbf{i}}=-\left(\varphi_{i_{i}^{\prime}}-\psi_{0}\right)
$$

Cet écart initial peut provenir par exemple d'une variation de l'intensité débitée par la batterie. Jusqu'au moment $t=0$, la batterie débitait un certain courant et la tension aux bornes avait la valeur normale $p_{\mathrm{n}}$. Au moment $t=0$, l'intensité est modifiée par le fonctionnement d'un interrupteur et la tension s'élève subitement de $\Delta_{\mathrm{i}}$. C'est à ce moment-là qu'intervient le régulateur, pour ramener la tension à sa valeur de consigne.

En tenant compte de la condition' 11 , les deux équations deviennent :

$$
\begin{gathered}
\left(\psi-\psi_{0}\right)=\left(\psi_{1}-\psi_{0}\right) e^{-\frac{\partial}{\mathrm{T}_{\mathrm{S}}} t} \\
\Delta=\Delta_{\mathrm{i}} e^{-\frac{\gamma}{\mathrm{T}_{\mathrm{s}}} t}
\end{gathered}
$$

L'écart initial $\Delta_{i}$ est donc ramené à zéro suivant une loi exponentielle. Le signe de $\gamma$, c'est-à-dire le sens des connexions de la batterie au cadran est défini par le signe de l'exposant. La traduction graphique de cette équation est représentée par la fig. 10.
La correction dure, on le voit, en principe un temps infiniment long. En pratique, le réglage est terminé dès que l'écart $\Delta$ est devenu égal à l'insensibilité du régulateur.

L'opération de réglage est donc stable ; des oscillations ne sont pas à craindre. Toutes choses égales d'ailleurs, le réglage cst terminé d'autant plus rapidement que la durée spécifique de parcours du régulateur $T_{s}$ est plus petite, ou que la répartition des éléments sur le cadran est plus concentrée ( $\gamma$ grand).

\section{Réglage de la tension d'une génératrice actionnée à vitesse constante.}

\section{Premier Cas : Excitation a tension constante}

Etablissement de l'équation de la machine. -- Dans une génératrice à courant continu ou alternatif, à excilation séparée, la tension aux bornes peut s'exprimer par: $\mathrm{P}=\mathrm{E}-\mathrm{IX}$.

$E$ étant la force électromotrice,

I l'intensité débitéc.

$\mathrm{X}$ une impédance intérieure traduisant la chute de tension due au débit de la maohine.

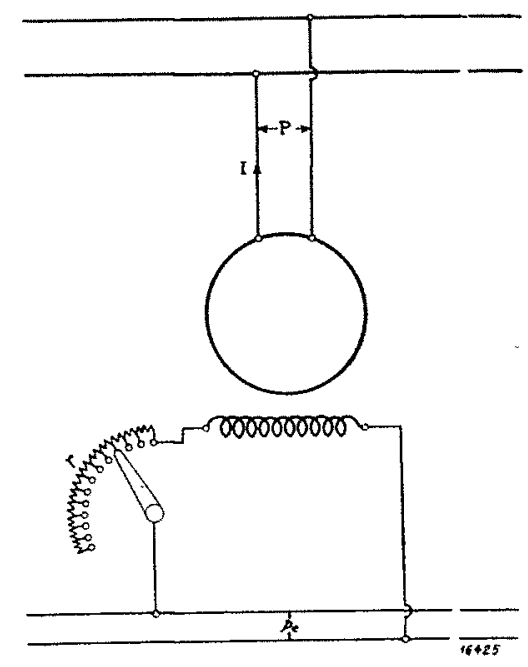

Fig. 16.

Cette équation étant linéaire, les variatiı ns de courant I produisent des variations proportionnelles de la tension aux bornes $P$, et ces variations devront être corrigées par des variations égales de la force électromotrice, c'est-à-dire du flux inducteur. Nous supposons donc toujours, pendant la durée de la correction, l'intensité (la charge) invariable, et nous avons donc à examiner comment la tension aux bornes ou, ce qui revie it au même, comment la force électromotrice variera pendant l'opération de réglage. Pendant le temps qui précédait la perturb xtion, la tension avait sa valeur normale de consigne, et nous avi ins :

$$
\mathrm{P}_{\mathrm{n}}=\mathrm{E}_{1}-\mathrm{I}_{1} \mathrm{X}
$$

A un certain moment, qui constitue pour nous l'origine des temps, l'intensité prend brusquement une valeur différente et nous avons :

$$
P_{2}=E_{1}-I_{2} X
$$

L'écart de tension $P_{2}-P_{n}$ doit donc êt e corrigé par une modification de la force électromotrice qui presıdra la valeur finale $\mathrm{E}_{2}$ telle qu'on ait de nouveau :

$$
\mathrm{P}_{\mathrm{n}}=\mathrm{E}_{2}-\mathrm{I}_{2} \mathrm{X}
$$

La différence $P_{2}-P_{n}$ ou son égale $E_{2}-E_{1}=\Delta E$ est l'écart 
initial de tension, et le quotient $\frac{\Delta E}{P_{n}}=\Delta_{i}$ est notre écart initial relatif.

A chaque instant de l'opération de réglage, nous avons : $\mathrm{P}=\mathrm{E}-\mathrm{I}_{2} \mathrm{X}$, et l'écart $\mathrm{P}-\mathrm{P}_{\mathrm{n}}$ ou son égal, pris relativemenl $\frac{\Delta \mathrm{E}}{\mathrm{P}}=\perp$ doit donc être ramené à zéro par le jeu du régulateur.

Mais pour modifier la force électromotrice nous agissons sur le courant d'excitation. Nous avons donc à rechercher la relation qui lic l'écart de force électromotrice $\Delta \mathrm{E}$ à la valeur de la résistance d'excitation, par l'intermédiaire du courant d'excitation.

Or, Iorsqu'on modifie la position, c'est-à-dire la valeur de la résistance d'excitation, le courant d'excitation varie suivant la loi :

$$
\mathrm{L} \frac{d i}{d t}+i r=p_{\mathrm{e}}
$$

ou 1. -.. coefficient de selfinduction du circuit inducteur,

$r=$ résistance tolale du circuit d'excitation,

$p_{\mathrm{e}}=$ Lension d'excitalion.

équalion qui s'écril aussi :

$$
\frac{\mathrm{I}}{r} \frac{d i}{d t}+i=\frac{p_{\mathrm{e}}}{r}
$$

Or, $\frac{\mathrm{L}}{r}=$ T est la constante de temps du circuit d'excitation Cette valeur n'est en réalité pas constante d'abord puisque $r$ varic puis, parce que le coefficient de selfinduction des inducteurs varie avec la perméabilité, c'est-à-dire avec le courant d'excitalion lui-même. Mais on peut considérer le quotient comme conslant et calculer avec une moyenne, au moins dans une certaine ctendue. La variation de $L$, due à la perméabilité, se faisant dans le mème sens que la variation de $r$, il se produit, pour le quotient, une certaine compensation. En outre, l'introduction d'une fonction variable pour cette constante de temps rendrait le calcul si compliqué qu'il devient impossible de dominer les formules et d'en lirer des résultats pratiques.

Nous écrivons donc :

$$
\mathrm{T} \frac{d i}{d t}+i=\frac{p_{\mathrm{e}}}{r}=i_{\mathrm{s}}
$$

Lo quolient $\frac{p_{s}}{r}=i_{\mathrm{s}}$ représente la valeur stationnaire que prend le courant d'excitation lorsque la résistance du circuit est égale à $r$. Celte valeur stationnaire définit donc sans ambiguïté la résistance du circuit d'excitation et la position du curseur.

Reste à établir la relation entre le courant d'excitation et la force électromotrice de la machine. Dans la région dans laquelle travaille une génératrice à tension constante, la caractéristique peul, avec unc approximation bien suffisante, être remplacée par une dépendance linéaire du courant d'excitation.

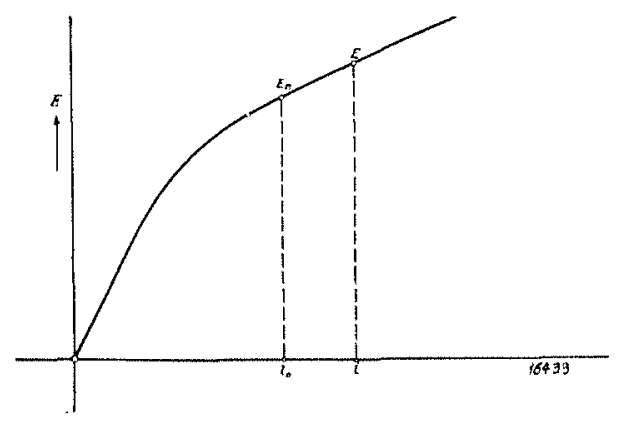

lig. 17.

En choisissant donc comme référence le courant d'excitation $i_{0}$ auquel nous devons arriver pour rétablir la force électromotrice, et par conséquent la tension aux bornes, à sa valeur de consigne, nous aurions :

$$
\Delta=\frac{\mathrm{E}-\mathrm{E}_{\mathrm{n}}}{\mathrm{P}_{\mathrm{n}}}=m \cdot\left(i-i_{0}\right)
$$

et, par conséquent, en introduisant dans 14)

$$
\mathrm{T} \frac{d \Delta}{d t}+\Delta=\Delta_{\mathrm{s}}
$$

où $\Delta_{\mathrm{s}}$ est l'écart relatif obtenu en régime stationnaire qui correspond à la position du rhéostat d'excitation. $\Delta_{s}$ se mesure par l'écart d'avec la tension normale, rapporté à cette dernière en \%. qu'on lirait au voltmètre si on laissait au régime le temps de redevenir stationnaire. $\Delta_{s}$ caractérise donc sans ambiguïté la position du curseur du rhéostat d'excitation, c'est-à-dire le paramètre de réglage.

Cette équation 16 est l'équation de la machine en régime perturbé. En modifiant la position du rhéostat d'excitation c'est-à-dire la valeur de $\lrcorner_{s}$, on produit une modification de la tension, et par suite, de l'écart $\lrcorner$ actuel d'avec la tension de consigne. La modification de $\Delta_{\mathrm{s}}$ se fait par le jeu du régulateur automatique soumis à l'information de l'écart momentané.

\section{Equation de la Corrrection}

Le régulateur automatique soumis à l'information de l'écart provoque donc un déplacement de son arbre de réglage suivant la loi (éq. 5).

$$
\frac{d \Delta}{d t}+\frac{\Delta}{\mathrm{T}_{\mathrm{a}}}=\mathrm{T}_{\mathrm{s}} \frac{d^{2} \downarrow}{d t^{2}}+\left(\grave{s}+\frac{\mathrm{T}_{\mathrm{s}}}{\mathrm{T}_{\mathrm{a}}}\right) \frac{d \downarrow}{d t}
$$

D'autre part, le déplacement du curseur du rhéostat caractérisé par la variation de l'écart stationnaire produit une modification de l'écart momentané selon la loi (éq. 16).

$$
\frac{d \Delta}{d t}+\frac{\Delta}{\mathrm{T}}=\frac{\Delta_{\mathrm{s}}}{\mathrm{T}}
$$

Comme le curseur du rhéostat est commandé par l'arbre du rẻgulateur automatique, nous avons donc entre le déplacmeent ". du régulateur et la valeur $\Delta_{\mathbf{s}}$ du paramètre de réglage, c'est-à-dire du rhéostat d'excitation, une relation qui dépend du mode d'entraînement, ou si l'on veut, de la répartilion des résistances sur l'appareil à touches. Cette répartition, nous l'admettons linéaire ; c'est la seule qui donne les mêmes conditions de correction sur toute l'étendue d'utilisation de la machine. Nous poserons donc :

$$
\Delta_{\mathrm{s}}=-\because\left(\psi-\psi_{0}\right)
$$

On voit que $\Delta_{\mathrm{s}}=\mathrm{O}$ pour $\psi=\psi_{0} . \psi_{0}$ est donc la position du curseur du régulateur pour laquelle l'écart stationnaire est nul : c'est donc l'endroit où devra stationner le curseur du régulateur une fois que la correction sera terminée.

$\gamma$ est ici de nouveau le coefficient de répartition. Son signe dépend évidemment du sens des connexions du rhéostat et est parfaitement déterminé par le fonctionnement du régulateur. Il représente l'écart total de tension entre les deux extrémités"de la course du régulateur.

L'équation de la correction résulte donc du système des deux équations existant simultanément :

$$
\begin{aligned}
& \frac{d \Delta}{d t}+\frac{\Delta}{\mathrm{T}_{\mathrm{a}}}=\mathrm{T}_{\mathrm{s}} \frac{d^{2} \mathrm{U}}{d t^{2}}+\left(\delta+\frac{\mathrm{T}_{\mathrm{s}}}{\mathrm{T}_{\mathrm{a}}}\right) \frac{d U}{d t} \\
& \frac{d \Delta}{d t}+\frac{\Delta}{T}=-\frac{i}{\mathrm{~T}}(\dot{\varphi}-\hat{u})
\end{aligned}
$$

En éliminant $\psi$, nous obtiendrons l'équation de l'écart $\Delta$ de la tension, et en éliminant $\lrcorner$, nous aurons le parcours $\downarrow$ du régulateur. 


\section{ASSERVISSEMENT CONCORDANT}

Remarquons que les membres de gauche des deux équations ne diffèrent que par les constantes de temps $\mathrm{T}$ de la machine et $T_{a}$ u régulateur. Jusqu'ici nous n'avons disposé d'aucun coefficient d, régulateur. Rien ne s'oppose à ce que nous donnions à $T_{a}$ une valeur particulière. Nous choisirons donc arbitrairement :

$$
\mathrm{T}_{\mathrm{a}}=\mathrm{T}
$$

c'est-à-dire : constante de temps asservissement $=$ constonte de temps inducteurs et établirons le résultat dans cette hypothèse. Nous montrerons ensuite comment ce résultat se medifie si $T_{a}$ diffère de $T$, ce qui se produit en particulier parce que T n'est pas absolument invariable, et nous verrons que cette condition d'égalité correspond à la loi de correction la plus favorable.

L'élimination de $\lrcorner$ et de ses dérivées, dans cette hypothèse, nous donne, pour le déplacement de l'organe de réglage, l'équation :

$$
\frac{d^{2} \psi}{d t^{2}}+\left(\frac{\partial}{T_{s}}+\frac{1}{T}\right) \frac{d \dot{u}}{d t}+\frac{\ddot{i}}{\mathrm{~T}_{\mathrm{s}} \mathrm{T}}\left(\dot{u}-\psi_{0}\right)=0
$$

Remarquons enfin que $\psi_{0}$ étant par définition la posilion que prendra l'organe de réglage une fois l'état stationnaire atteint, $\left(\triangleleft-\psi_{0}\right)$ est donc l'écart entre la position actuelle et cette position finale. En rapportant le mouvement à cette position finale comme origine, nous pouvons donc considérer l'écart momentané $x=\left(\downarrow-\omega_{0}\right)$ comme variable et écrire notre équation sous la forme :

$$
\frac{d^{2} x}{d t^{2}}+\left(\frac{\partial}{\mathrm{T}_{\mathrm{s}}}+\frac{1}{\mathrm{~T}}\right) \frac{d x}{d t}+\frac{\gamma}{\mathrm{T}_{\mathrm{s}} \mathrm{T}} x=0
$$

\section{Intégration des Equations pour le Cas d'ten Réglilatelr} DIT " Rapide"

L'intégration de cette équation ne présente pas de difficulté ; c'est, comme on le sait, l'équation d'une oscillation amortie, tous les coefficients étant positifs. Toutefois nous nous limiterons au cas des régulateurs dits rapides, c'est-à-dire de ceux dans lesquels la durée spécifique de parcours $T_{s}$ est très petite comparativement à la constante de temps $T$ des inducteurs. A titre d'orientation nous remarquerons que $T_{s}$ a pour le régulateur à huile des Ateliers Cuénod, la valeur $\mathrm{T}_{\mathrm{s}}=0,02$ " et $\delta$ est en général compris entre 0,5 et 1,5 , alers que la constante de temps d'une petite machine de $20 \mathrm{kw}$ atteint déjà $T=0,5$ " et peut arriver à 4" pour un alternateur de $10.000 \mathrm{kVA}$.

Dans cette hypothèse, la solution générale de l'équation est :

$$
x=\mathrm{C}_{1} e^{-\frac{t}{2 \mathrm{~T}}}+\mathrm{C}_{2} e^{-\left(\frac{\delta}{\mathrm{T} s}+\frac{1}{2 \mathrm{~T}}\right) t}
$$

\section{Conditions aux Limites}

Les constantes d'intégration se déterminent par les considérations suivantes :

Au début du réglage, c'est-à-dire pour $t=0$, le curseur occupe la position initiale $w_{\mathrm{i}}$ ou $x_{\mathrm{i}}$ - Donc pour $t=0, x=x_{\mathrm{i}}$.

D'autre part, la perturbation étant supposée succéder à un état stationnaire, le régulateur était immobile et la lame d'asservissement en particulier, occupait sa position de détente complète : $u=0$. Il en résulte, par les équalions 4) et 5)

$$
\frac{d z}{d t}=\mathrm{V} y=\frac{2 M i_{n}^{2} \Delta_{\mathrm{I}} \mathrm{V}}{\beta}=\frac{\Delta_{\mathrm{i}} \mathrm{S}}{\mathrm{T}_{\mathrm{s}}}
$$

en tenant compte de la signification de $\mathrm{T}_{\mathbf{s}}$.
$\Delta_{1}$ est l'écart de lension initial, créé par la perturbation de charge et qui constituc le début de l'opération de réglage. $\Delta_{\mathbf{i}}$ est du reste lié à $x_{i}$ par l'équation 18 , c'est-à-dire :

$$
\Delta_{\mathrm{i}}=-\gamma x_{\mathrm{i}}
$$

ce qui donne pour la deuxième condition limile :

$$
\text { pour ; } t=0 \quad \frac{d x}{d t}=-\frac{\gamma x_{\mathrm{i}}}{\mathrm{T}_{\mathrm{s}}}
$$

En introduisant ces deux conditions dans l'équation nous en déterminons les constantes et l'équation devient, après simplification :

$$
x=x_{\mathrm{i}}\left\{\frac{\gamma}{\delta} e^{-\frac{\hat{\sigma}}{\mathrm{T} \mathrm{s}} t}-\left(\frac{\gamma}{\delta}-1\right) e^{-\frac{t}{2 \mathrm{~T}}}\right\}
$$

\section{Discussion de cette Equation}

En pronant le lemps $t$ comme abscisse d'un système reclangulaire el comme ordonnée le déplacement $x$ du curseur, l'origine étant la position que le curseur occupera une fois l'opération de réglage terminée, l'équation ci-dessus se traduit par la courbe reprèsentéc par fig. 18. On voit qu'au moment où la perturbation

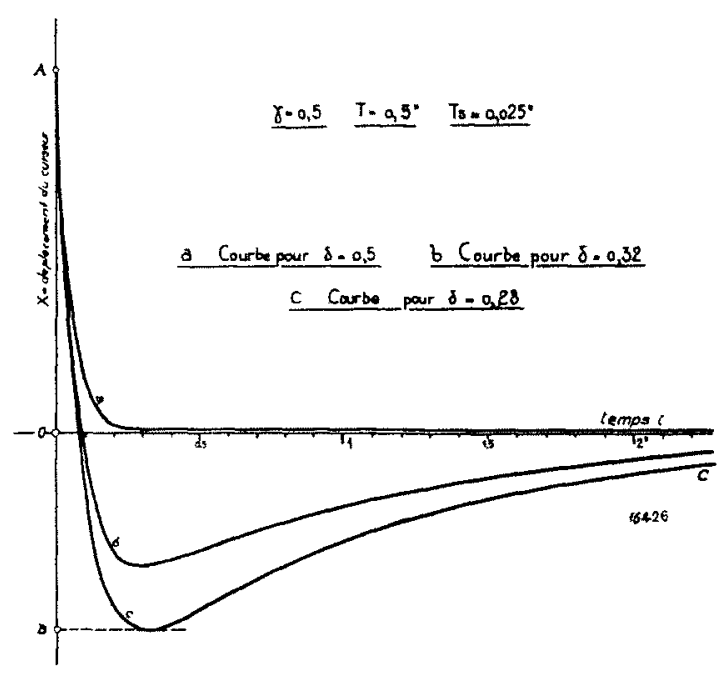

Fïg. 18.

se produit, le curseur se précipite en quelque sorte vers la position $O$ qu'il devra occuper, la dépasse jusqu'à un certain minimum $B$, pour remonter ensuite beaucoup plus lentement vers l'axe des abscisses, c'est-à-dire vers sa position finale. Ce dépassement $\mathrm{OB}$ force en quelque sorte le courant d'excitation à se développer plus rapidement. Il correspond donc au surréglage que tout élertricien opère d'instinct à la main. On voit en parliculier quelle est l'importance d'un rhéostat de champ suffisamment étendu, c'està-dire en général, d'une marge d'action suffisamment vaste pour le paramètre de réglage.

La partie ascensionnelle B C, qui s'opère beaucoup plus lentement, provient essentiellement du terme en $e^{-\frac{t}{2 \mathrm{~T}}}, c^{\prime} \operatorname{csl}-\dot{\mathrm{a}}$-dire de la constante de temps $\mathrm{T}$ des inducteurs.

Si A représente par cxemple la position de pleine charge de la machine et $O$ la position de marche à vide, on peut se rendre compte de combien le rhéostat doit être prolongé de chaque côté pour que le réglage s'opère dans les meilleures conditions.

Le minimum $B$ dépend essentiellement des valeurs du décrément d'asservissement $\partial$. La fig. 18 donne les différentes courbes du déplacement du curscur pour une même machine et un même rhéostat avec différentes valeurs de s. 


\section{AIJURE DE LA TENSION PeNDANT L'OpÉRATION DE RÉglage}

Comnaissant la valeur de $x$, il est aisé de calculer l'allure de l'écart $\Delta$ de la tension par l'équation 20 .

La constante d'intégration se détermine par la condition limite de l'écart initial. A l'instant qui suit immédiatement la perturbation, l'écart de tension est $\Delta_{1}$ et constitue le début de l'opération de réglage. Au reste, $\Delta_{i}$ est lié à la position iniliale du curseur par l'équation 18 , c'est-à-dire :

$$
\Delta_{\mathbf{i}}=-\gamma x_{\mathbf{i}}
$$

Inlégréc avec les mêmes simplifications que précédemment, relatives à $T_{s}$, l'équation devient:

$$
J=\Delta_{\mathrm{i}}\left\{\left(\frac{2 y}{\partial}-1\right) e^{-\frac{t}{\mathrm{~T}}} \lambda-\left(\frac{2 y}{\partial}-2\right) e^{-\frac{t}{2 T}}\right\}
$$

Ce résultat est remarquable par la circonstance qu'il ne contient plus aucun terme en $\mathrm{T}_{\mathrm{s}}$; l'écart de tension est donc com litcment indépendant de la durée spécifique du parcours $T_{s}$. Les deux exponentielles, fonction uniquement de la constante de temps des inducteurs, disparaîtront donc dans un temps d'autant plus long que la constanle de temps sera plus élevée. Aucune amélioration dans la durée de la correclion ne peut être apportée par une augmentation de la vilesse du régulateur. On peut donc énoncer le résultal sous la forme suivante : la durée de la correction de la tension, pour autant que celte correction est effectuée par un régulateur rapide ( $\mathrm{T}_{\mathrm{s}}$ petit à rôté de $\mathrm{T}$ ) ne dépend plus du régulateur, mais uniquement de la machine réglée. Cette conclusion s'applique à tous les régulateurs dont le fonctionnement est traduit par l'équation 5), pratiquement à tous les régulateurs rapides existant actuellement.

Ici se pose donc, pour le constructeur de machine, une nouvelle exigence, relative à la constante de temps. Ce n'est qu'en réduisant cette valeur qu'il pourra avec succès faire usage des machine à forte chute de tension combinées avec régulateur automatique.

$$
\text { En portant en }
$$
abscisse le temps et en ordonnée l'écart, la courbe de correction a l'allure représentée par fig. 19. Les différentes courbes diffèrent les unes des autres par la valeur du déerément $\delta$. On remarque en particulier qu'en augmentant le décré-

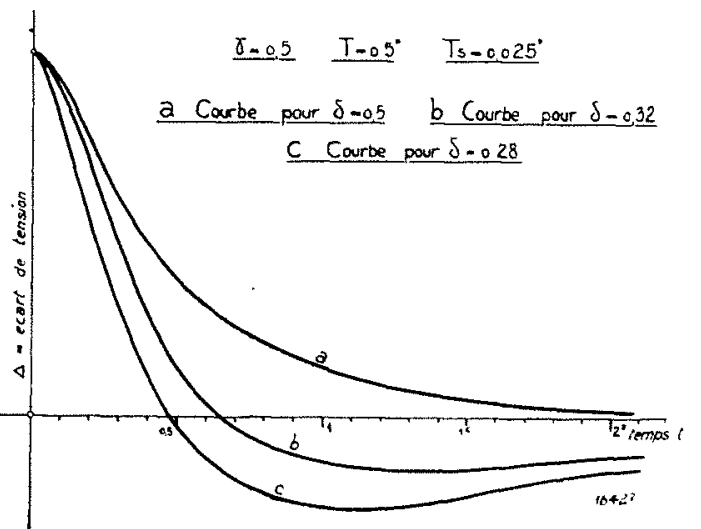

Fig. 19. le graphique suivant : la tension est constante jusqu'au momen où survient une variation de charge : soit A l'une d'elles (fig. 20).

Fig. 20 .

Au moment où la variation d'intensité se produit, la tension s'élève (ou s'abaisse) d'une valeur initiale $\lrcorner p_{i}$. Puis, sans perdre un instant, le régulateur intervient et ramène la tension à sa valeur normale, dans un temps qui ne dépend essentiellement que de la machine elle-même. Cette variation de tension initiale se produit inexorablement à chaque variation d'intensité. Il est donc absurde de prétendre qu'un régulateur automatique puisse maintenir continuellement la tension dans les limites de son insensibilité. Il l'y ramène, dans un temps plus ou moins long, mais il est impuissant contre l'écart initial. Il est juste cependant de constater que souvent les voltmètres enregistreurs ne suivent pas toujours l'écart, du moins dans toute son élongation, ce qui fait croire à tort à la rapidité d'action du réglage.

Les enregistreurs déroulant en général leur papier très lentement, toute la correction se confond en une seule ordonnée et le diagramme apparaît sous forme d'un trait plus ou moins épais, selon que le régime de la machine est plus ou moins tourmenté.

\section{ASSERVISSEMENT DISCORDANT}

Nous avons posé précédemment: $\mathrm{T}_{\mathrm{a}}=\mathrm{T}$.

Il est facile de se rendre compte de l'influence d'une différence entre la constante de temps de l'asservissement et celle desi nducteurs.

$1^{\text {o }}$ Supposons $\mathrm{T}_{\mathrm{a}}>\mathrm{T}$. La détente de la lame d'asservissement reste en arrière sur le développement du courant d'excitation et la correction se ralentit. Comme cas limite, envisageons $T_{a}=\infty$ ce qui revient à remplacer le frein à huile par une tige rigide. Lorsque le réglage sera terminé, la tension n'aura plus sa valeur de consigne, mais restera en permanence ou plus petite ou plus grande, selon le sens de la variation. Si $T_{\mathrm{a}}$ sans être $\infty$, est très grand, la tension reprendra sa valeur normale, mais excessivement lentement. La durée de la correction se trouve donc augmentée.

$2^{\circ}$ Supposons $\mathrm{T}_{\mathrm{a}}<\mathrm{T}$, et poussons à l'extrême $: \mathrm{T}_{\mathrm{a}}=0$, ce qui revient à supprimer l'asservissement. On se rend aisément compte, en résolvant les équations dans cette hypothèse que la tension devient oscillatoire, d'amplitude décroissante. Elle passe donc plusieurs fois par sa valeur de consigne avant de se stabiliser, ce qui évidemment est un inconvénient.
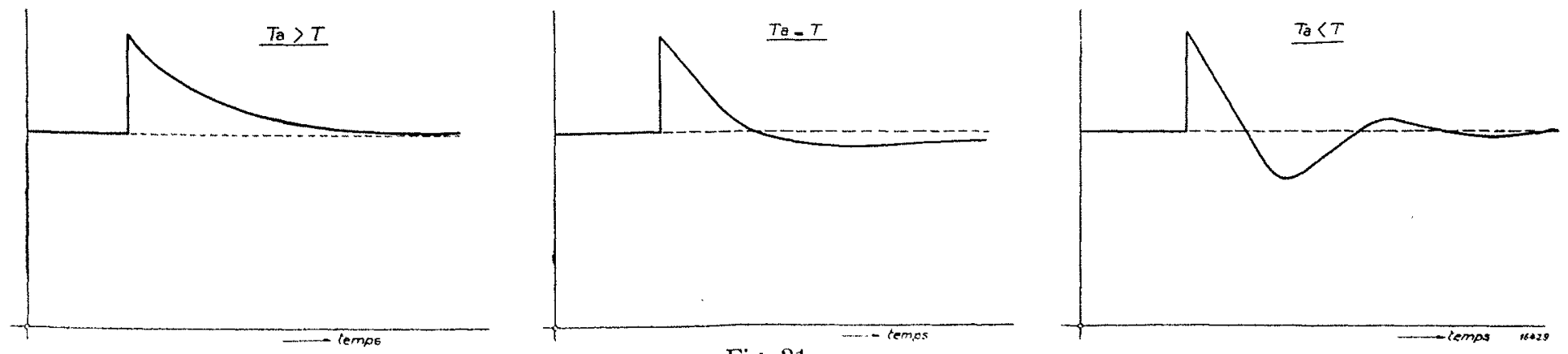

Fig. 21.

ment, on retarde le moment où la tension a repris une valeur comprise entre deux limites données $\pm \varepsilon$. Par contre, on limite égalcment la pointe de sens inverse $B$; la valeur optimum de $\delta$ est ici d'environ 0,3 .

Le régime général de notre généralrice se représente donc par
Les trois cas sont donc représentés par la figure 21.

Il y a donc tout intérêt à réaliser $T_{a}=T$, c'est-à-dire l'égalité de la constante de temps de l'asservissement et de la constante de temps moyenne des inducteurs.

(A suivre). 